\title{
QUANTITATIVE CHARACTERIZATION OF MULTICOMPONENT POLYMERS BY SAMPLE CONTROLLED THERMAL ANALYSIS
}

\section{Pedro E. Sánchez-Jiménez ${ }^{*, a}$, Luis A. Pérez-Maqueda ${ }^{a}$, José E. Crespo-Amorós ${ }^{b}$, Juan López-Martínez ${ }^{\text {, Antonio Perejón }}{ }^{\text {a }}$ and José M. Criado ${ }^{\text {a }}$.}

a. Instituto de Ciencia de Materiales de Sevilla, C.S.I.C.-Universidad de Sevilla, C. Américo Vespucio n49, 41092 Sevilla, Spain

b. Department of Mechanical and Materials Engineering, Polytechnic University of Valencia, 03801 Alcoy-Alicante, Spain

\begin{abstract}
This paper explores the potential of Sample Controlled Thermal Analysis (SCTA) in order to perform compositional analysis of multicomponent polymeric materials by means of thermogravimetric experiments. In SCTA experiments, the response of the sample to the temperature determines the evolution of the temperature by means of a feedback system, thus, what is controlled is not the temperature-time profile as in conventional analysis, but rather the evolution of the reaction rate with time. The higher resolving power provided by the technique has been used for determining the composition of polymer blends composed of polyvinyl chloride (PVC) and different commercial plasticizers, a system where the individual components have very similar thermal stabilities, thereby rendering useless thermogravimetric experiments run under conventional conditions. Different SCTA procedures such as Constant Rate Thermal Analysis (CRTA), which has received special attention, Hi-Res and Stepwise Isothermal Analysis have been tested and the results obtained have been compared with linear heating rate technique. It has been proven that CRTA can be used to effectively determine the exact composition of the blend.
\end{abstract}

\footnotetext{
${ }^{*}$ Corresponding author. Tel +34954489548 Fax +34954460665

e-mail address: pedro.enrique@icmse.csic.es
} 


\section{Introduction}

Polymers are one of the most important types of materials in both functional and structural applications. The fact that they are easily modified by including additives in the formulation or by preparing copolymers in order to tailor their properties grants them a flexibility that contributes greatly to their ever-increasing technological importance. The compositional analysis of complex polymers is usually performed by means of extraction and chromatographic techniques. ${ }^{1-4}$ However, those techniques can be time consuming and complex. Thermal analysis (TA) techniques are routinely used for thermal characterization in laboratories and industries that work in the manufacture and development of polymers and constitute an interesting alternative for analytical studies as they feature both sensitivity and simplicity. Among TA techniques, thermogravimetry (TG) can be used for precise and accurate compositional analysis, and for identification of polymers from their decomposition pattern, and therefore can be used for quality and process control. ${ }^{1,5-6}$ This fact is illustrated by the ASTM E1131 norm that has been developed in order to describe a standard method of compositional analysis by thermogravimetry. ${ }^{7}$ Thus, if a multicomponent polymer material is composed of blocks with different thermal stabilities, it is possible to quantitatively analyze them by TG. ${ }^{1,7}$ Two classical examples are the quantification of the copolymer polyethylene-co-(vinyl acetate) (EVA) and the compositional analysis of polymeric vulcanized rubber that has been used for many years to determine the quality and content of various rubber products. ${ }^{6-7}$

The main disadvantage of TG for analytical studies is the requirement for the different components to have different thermal stabilities. In the case of overlapping processes that happen at similar temperature ranges, the applicability of TG for quantitative analysis is severely limited. Attempts to improve the ability to separate closely occurring event, by changing the heating rate in conventional heating schedules might yield better results, but are not always sufficient for a proper discrimination. This is the case with plastisols, composed of mixtures of vinyl resins, mainly PVC, and added plasticizers that enhance polymer flexibility and improve the processing. Phthalate esters have been used as plasticizers to prepare flexible PVC products since the $1920 \mathrm{~s}^{8-}$ ${ }^{10}$ but in recent years many studies have reported possible toxic effects of some of the phthalates on human health and on the environment. ${ }^{11-15}$ As a consequence, several 
papers dealing with phthalate plasticizer migrations and extractions have been published recently. ${ }^{11,16-21}$ The determination of plasticizer content is performed by extraction, chromatographic techniques, mass spectrometry, infrared spectroscopy, and NMR spectroscopy. ${ }^{1-2,11,22-24}$ While some studies have applied standard TA techniques to the study of plastisols, so far the proper discrimination between both components has not yet been resolved as the decomposition of the PVC overlap with the release of the plasticizer. $^{25-29}$

Sample Controlled Thermal Analysis (SCTA) constitutes an alternative approach which provides a much higher resolving power and, therefore, is able to successfully overcome the shortcomings of conventional linear heating rate experiments regarding the separation of closely occurring decomposition events. In thermal analysis, the resolving power concerns the extent to which adjacent, or partially overlapping, thermally induced processes are separated. ${ }^{30}$ Despite this ability, the potential of SCTA for analytical studies has not been explored so far. In SCTA experiments what is controlled is not the temperature-time profile as in conventional analysis, but rather the evolution of the reaction rate with the time. ${ }^{30-35}$ Most usually, the temperature is controlled in such a way that the reaction rate is maintained at a constant value previously selected by the user along the entire process. In such a case, the technique is known as Constant Rate Thermal Analysis (CRTA). The enhanced control over the decomposition process provided by CRTA makes it a much more effective tool than conventional methods for the discrimination of overlapping processes. ${ }^{36-37}$ Furthermore, the temperature-time profile, which is not known in advance and depends on the evolution of the reaction with temperature, can provide a good deal of information about the nature of the process studied since it can be related with the mechanism that drives the reaction. ${ }^{38-40}$ Other widely used SCTA methods include Stepwise Isothermal Analysis (SIA) and the High Resolution TGA (Hi-Res) of TA instruments. In SIA, the sample is heated at a constant heating rate until the reaction rate exceeds a pre-set limit. At this point, the increase of temperature stops and the reaction proceeds isothermally until the rate becomes smaller than another pre-set limit, when the heating is resumed. ${ }^{41-42}$ In Hi-Res TGA, the heating rate is proportional to a function of the mass loss (or mass gain). Thus, when the reaction rate increases, the heating rate automatically decreases, providing a better resolving power for overlapping events. ${ }^{43-45}$ It should be noted that neither SIA nor HiRes techniques allow cooling. 
The aim of the work presented here is to highlight the potential of Constant Rate Thermal Analysis for the compositional analysis of multicomponent polymers. Towards that goal, a system composed of a commercial polyvinyl chloride (PVC) polymer modified with different commercial plasticizers was chosen as an example. The results provided by CRTA will be compared with those obtained by conventional heating rate procedures and both Hi-Res and SIA techniques.

\section{Experimental}

Plastisols were prepared mixing the PVC resin (PB $1172 \mathrm{H}$, ATOFINA, with a k value of 75) and the plasticizer by means of a K.A.PL, mod. 5KPMS (St. Joseph, Michigan, USA) mixer for five minutes at room temperature. The paste thus obtained was then treated in a vacuum chamber, model MCP mod. 001LC (HEK-GMBH, Lübeck, Germany), in order to remove the air, and then cured at $200{ }^{\circ} \mathrm{C}$ for nine minutes in a Carbolite furnace model 2416CG (Hope Valley, 533 GRB, UK).

Different plasticizers were used in this work, including Hexamoll DINCH (1,2Cyclohexane dicarboxylic acid, di-isononyl ester), Palatinol DOP (Di-(2-ethylhexyl) Phthalate), Palatinol DINP (Diisononyl Phthalate), Carboflex DPHP (Di(2-Propyl Heptyl) phthalate) and DOTP (Dioctyl Terephthalate). All of them were mixed in several proportions with pure PVC in order to prepare the blends for the study. Table 1 shows a summary of the samples studied here.

Thermal decomposition experiments were carried out with a TA Q5000 IR (TA instruments, Crawley, UK) thermobalance connected to a gas flow system to work in an inert atmosphere (70 cc $\mathrm{N}_{2}$ ). With this instrument, which has a TG signal sensitivity greater than $0.1 \mu \mathrm{g}$, experiments under conventional linear heating, constant rate thermal analysis (CRTA), stepwise thermal analysis (SIA) and Hi-Res TGA can be performed. 
Table 1. List of the different PVC-plasticizer blends that were prepared for this study.

\begin{tabular}{|c|c|c|c|}
\hline Sample & $\begin{array}{c}\text { PVC content } \\
\text { wt \% }\end{array}$ & $\begin{array}{c}\text { Plasticizers } \\
\text { content wt \% }\end{array}$ & $\begin{array}{c}\text { Plasticizer } \\
\text { used }\end{array}$ \\
\hline & & & \\
\hline PVC & 100 & 0 & - \\
\hline PVC-DINCH23 & 77.0 & 23.0 & DINCH \\
\hline PVC-DINCH34 & 66.5 & 33.5 & DINCH \\
\hline PVC-DINCH45 & 55.5 & 44.5 & DINCH \\
\hline PVC-DINP34 & 66.5 & 33.5 & DINP \\
\hline PVC-DOP34 & 66.5 & 33.5 & DOP \\
\hline PVC-DOTP34 & 66.5 & 33.5 & DOTP \\
\hline
\end{tabular}




\section{Discussion and Results}

Thermal degradation of PVC has usually been described as a two step process. The first step mainly involves the elimination of $\mathrm{HCl}$ and the simultaneous formation of sequences of conjugated double bonds in the polymer chain. At higher temperatures the material undergoes partial carbonization with the release of benzene and other aromatics. ${ }^{27}$ Plasticizers commonly used in industry evaporate at around $200^{\circ} \mathrm{C}$, although their inclusion in the polymeric matrix might change that temperature. ${ }^{27}$ In any case, the release of the plasticizer seems to take place in the same temperature range as the evolution of $\mathrm{HCl}$ due to the dehydrochlorination reaction, which would complicate any quantification by thermogravimetry measurements. This situation has already been reported by Jimenez et al., studying different plastisol samples, ${ }^{26}$ and by Brenes et al. ${ }^{28-}$ 29 when studying plastisized PVC samples used in cable insulation. In their studies, those authors confirm the overlapping of the plasticizer release and the dehydrochlorination, thereby rendering the discrimination of both components impossible under conventional linear heating experiments.

Figure 1 shows a typical mass loss versus time thermogravimetric curve obtained for the blend PVC-DINCH34 under a linear heating rate of $1 \mathrm{~K} \mathrm{~min}^{-1}$. In TG experiments the mass loss is a direct measure of the reacted fraction, while the derivative curve reflects the reaction rate. According to Figure 1, the blend is thermally stable up to $400 \mathrm{~K}$, at which point the dehydrochlorination process of PVC starts, finishing at around $600 \mathrm{~K}$. The curve has the typical sigmoidal shape and there is no indication of more than one process, as was expected. 


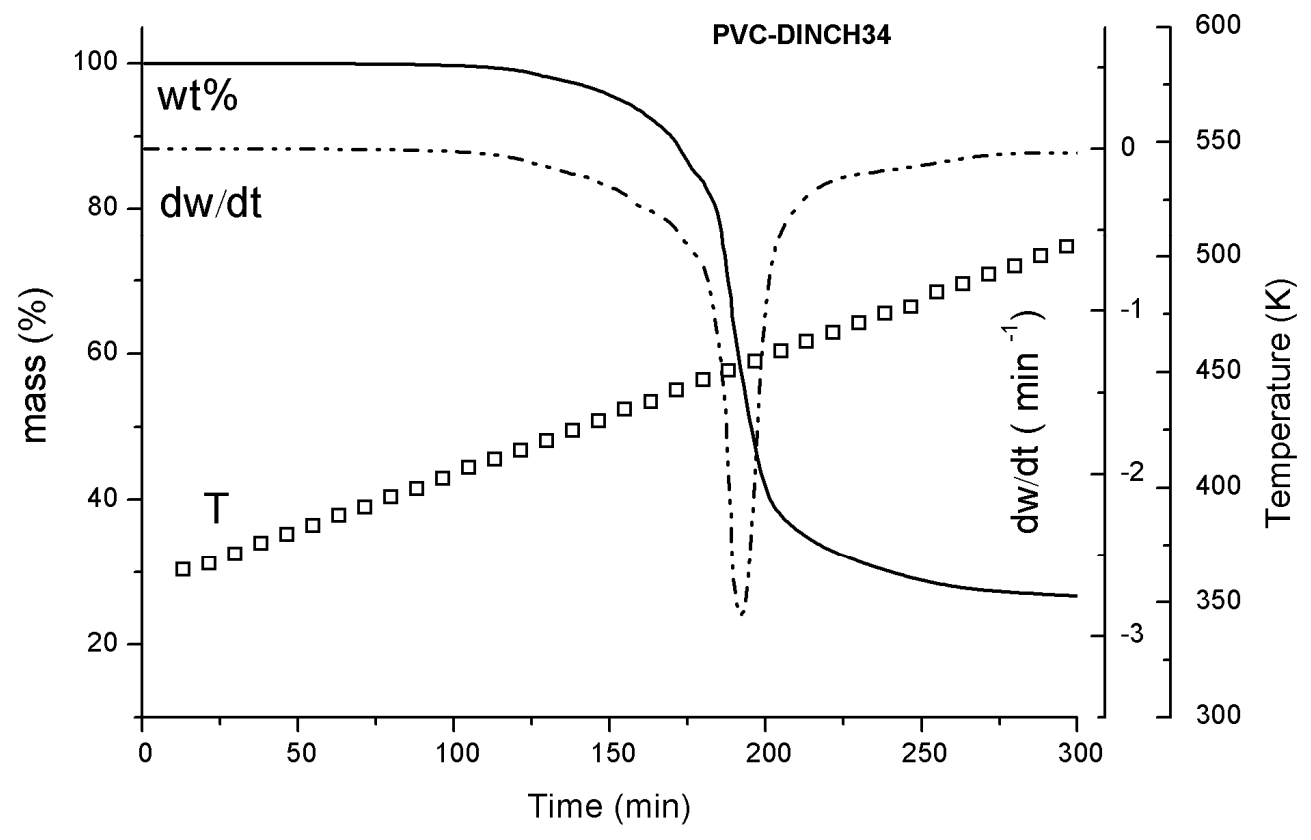

Figure 1. Experimental mass loss against time curve (solid lines) obtained for the thermal decomposition of the blend PVC-DINCH34 under a 90 cc $\mathrm{N}_{2}$ flow, and a linear heating rate of 1 $\mathrm{K} \mathrm{min}^{-1}$. The dotted line represents the derivative form of the degradation curve and the squared line represents the temperature profile. 
On the other hand, Figure 2 shows the mass loss, temperature and reaction rate as a function of time for the decomposition of the same PVC-DINCH34 blend under constant rate thermal analysis (CRTA) conditions. In order to properly compare the resolving power of both techniques, the mass loss rate was selected so that the total reaction time was approximately identical in both experiments. This was achieved by selecting a constant reaction rate of $0.003 \mathrm{~min}^{-1}$ for the CRTA run. It should be noted that identical scales have been used for Figures 1 and 2 in order to better stress the differences between both experiments. In the conventional linear heating experiment the evolution of the temperature with time follows a straight line, whose slope corresponds to the selected heating rate, while the mass loss has a typical sigmoidal shape. There is no feature in the curve that points out that two processes are involved. The main drawback of a linear heating temperature profile lies in the fact that very limited control over the reaction rate is achieved. Thus, the TG plot recorded at a heating rate of $1 \mathrm{~K}$ $\min ^{-1}$ shown in Figure 1 shows that the process is started and finished in a short 50 minute span. The main conclusion drawn is that a poor resolving power for overlapping processes can be expected from experiments performed under a linear heating profile even if very low heating rates are used. 


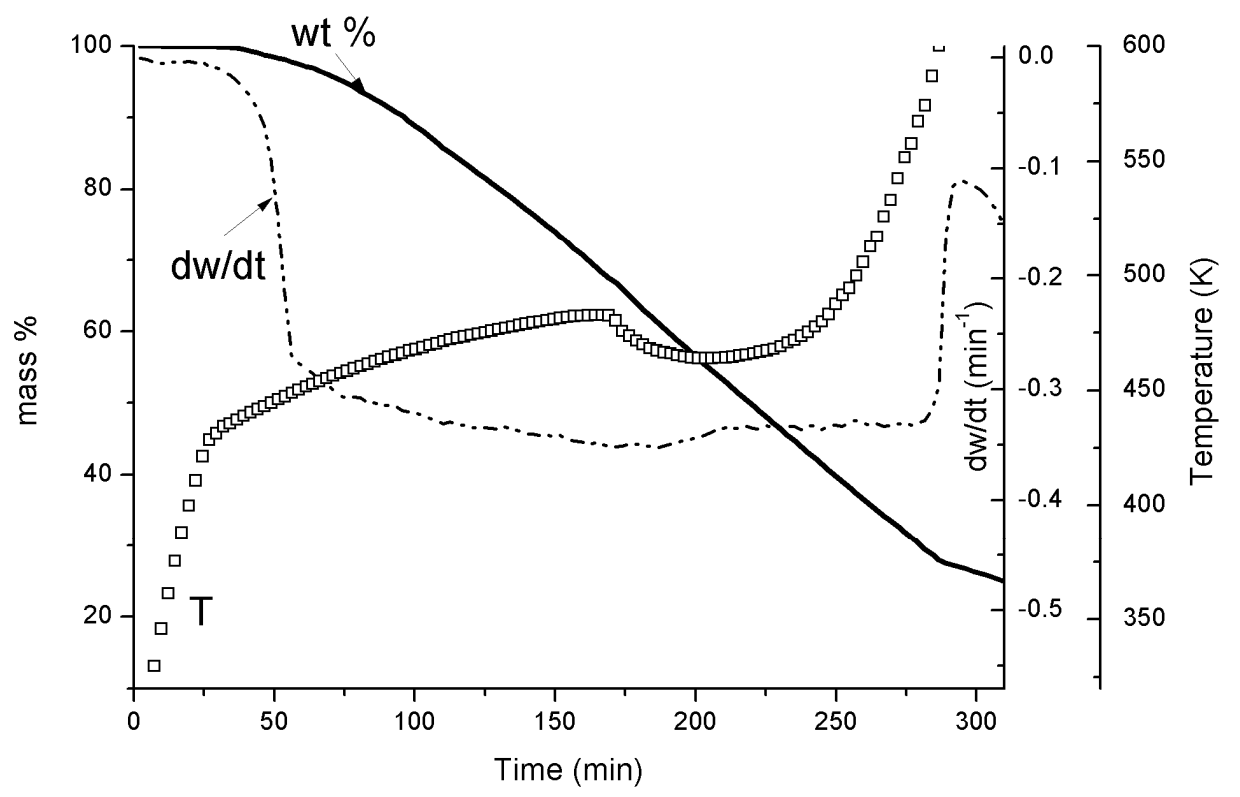

Figure 2. Experimental mass loss against time curve (solid line), obtained for the thermal decomposition of the blend PVC-DINCH34 under CRTA conditions. The degradation rate was set at a constant value of $0.003 \mathrm{~min}^{-1}$. The experiment was performed under an inert nitrogen atmosphere at a flow rate of 90 cc. The dotted line represents the rate of mass loss, and the squares correspond to the temperature-time profile. 
On the other hand, during the CRTA experiment (Figure 2), it can be observed how the reaction rate is maintained at an approximately constant value during the entire experiment. Unlike the experiment shown in Figure 1, under constant reaction rate conditions the existence of two processes stands out more clearly. The separation between them is marked by the crest in the temperature-time profile that appears at around $475 \mathrm{~K}$. The part of the curve before the crest corresponds to the evaporation of the plasticizers while the part of the curve beyond that point can be assigned to the dehydrochlorination of the PVC itself. The increased resolving power provided by the CRTA technique lies in its ability to keep the reaction rate under strict control. Here, there is no sharp increase in the reaction rate and the time elapsed since the start of the reaction until it finishes is five times longer than in the experiment run under conventional conditions (Fig 1). As a consequence, the plasticizer can be released completely before the dehydrochlorination actually starts. This feature can be observed more clearly when the mass loss is plotted against temperature. Figure 3 includes the CRTA mass-temperature curves obtained from the decomposition of the PVCDINCH34 blend (Fig. 3a) and from pure PVC (Fig. 3b), under identical experimental conditions. For the sake of comparison, Figure 3 also includes the decomposition curves for both the PVC-DINCH34 blend and pure PVC obtained under a linear heating profile of $1 \mathrm{~K} \mathrm{~min}^{-1}$. It can be noted from Figure 3 a that the maximum at the temperature versus mass loss that marks the end of the plasticizer release step appears after 33.5\% of the initial mass is lost, what matches closely with the amount of DINCH plasticizer that was included in the blend. It is also interesting to point out that the decomposition curves are displaced to much lower temperatures under CRTA conditions. Also, a careful look at the figure shows that the onset of the dehydrochlorination step appears at very much the same temperature in both the blend and in pure PVC. Therefore, it can be deduced that the decomposition of the PVC is unaffected by the inclusion of the plasticizer. Another interesting property of CRTA is that the evolution of temperature with time is not known in advance and it will depend on the kinetics of studied reaction. Thus, valuable information can be extracted from the shape of decomposition curves regarding the nature of the reaction ${ }^{38}$. It is then interesting to pay some attention to the differences between both curves. The temperature decrease that happens in the CRTA decomposition curve of the pure PVC decomposition curve just after the reaction starts can be related to the induction period that is characteristic of a nucleation driven 
process $^{38}$, which suggests such mechanism as the driving force for the dehydrochlorination step, as it has been recently reported in literature. ${ }^{46}$ 

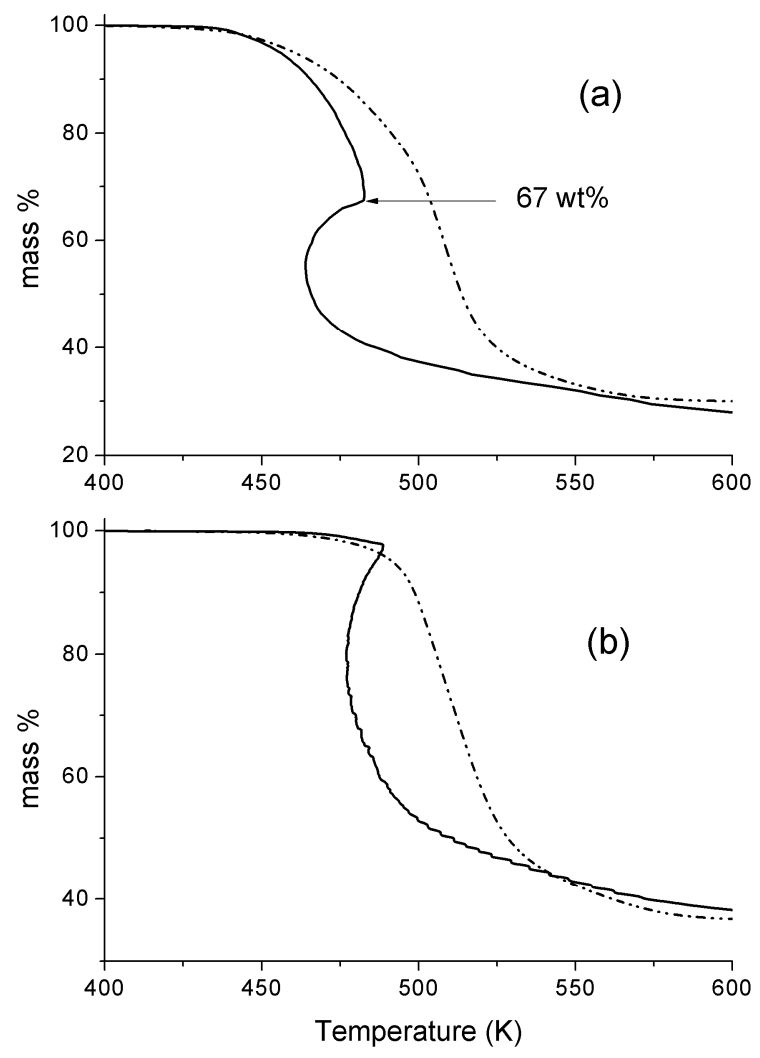

Figure 3. Experimental mass loss against temperature curve (solid lines) under CRTA conditions for the thermal decomposition of (a) a blend of PVC-DINCH34 and (b) pure PVC. A constant reaction rate of $0.003 \mathrm{~min}^{-1}$ and an inert atmosphere of $\mathrm{N}_{2}$ were maintained during the entire experiment. For the sake of comparison, experiments performed under linear heating rate (dotted lines) at $1 \mathrm{~K} \mathrm{~min}^{-1}$ have also been included. 
The identical feature that appears in the temperature profile can be found in the blend once the plasticizer is evolved, which confirms that point as the onset for $\mathrm{HCl}$ release. This information leads to the conclusion that the degradation of the polymer and that of the plasticizer are two independent processes as decomposition of the PVC does not seem to be significantly modified by the addition of the plasticizers. Regarding the release of the plasticizers, which happens just before the dehydrochlorination of the PVC, the shape of the CRTA curve corresponds to a diffusion controlled process. ${ }^{47}$ This mechanism is the one that has been proposed for the migration of the plasticizer in plastisols. $^{20}$

Figure 4 includes the mass loss-temperature curves obtained under controlled rate conditions for three blends (PVC-DINCH23, PVC-DINCH34 and PVC-DINCH45) with different plasticizer contents (23.0, 33.5 and 44.5 respectively) As expected, the "peak" in the temperature that signals the beginning of the dehydrochlorination step appears at a different percentage of mass loss depending on the amount of plasticizers included in the blend. In every case, the exact concentration of plasticizers can be determined directly from the curves. Alternatively, Figure 5 shows a set of mass loss-temperature CRTA curves obtained from the decomposition of four blends prepared using different plasticizers, but in equal content (PVC-DINCH34, PVC-DOP34, PVC-DOTP34 and PVCDINP34). In every case the decomposition curves obtained under conventional linear heating rate are also included for comparison. Again, only experimental runs performed under a constant decomposition rate are able to separate plasticizer release from the dehydrochlorination of PVC and, therefore, allow for the determination of the amount of plasticizers incorporated in every blend. 


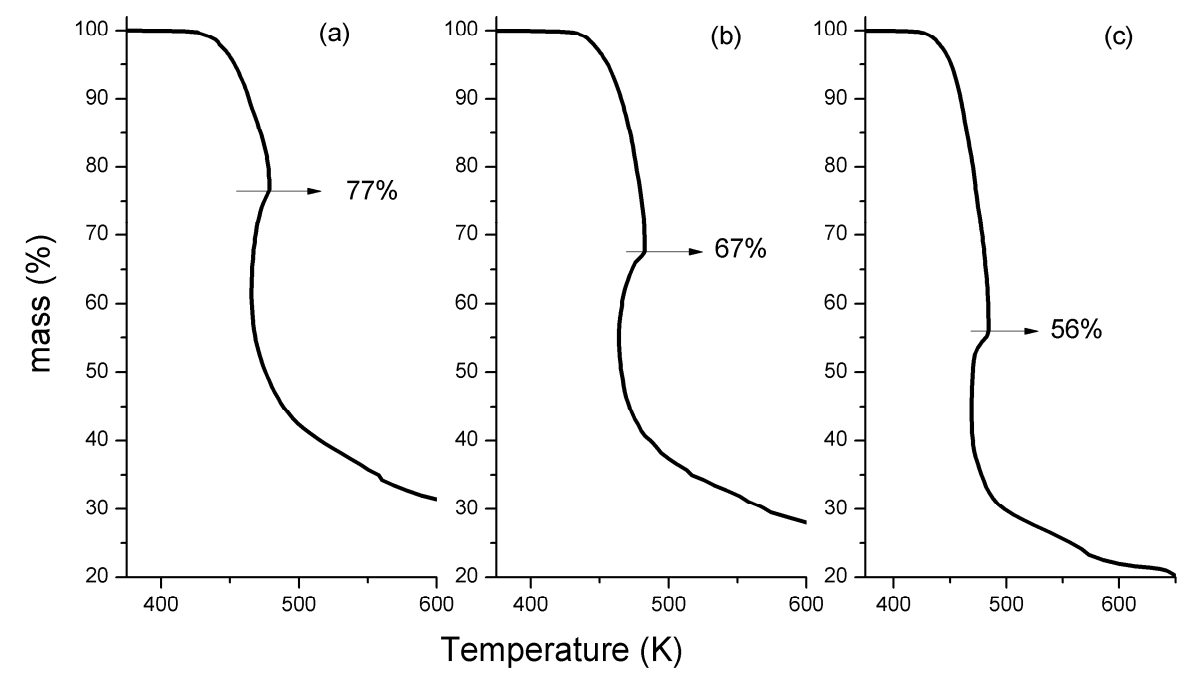

Figure 4. Experimental mass loss against temperature curve under CRTA conditions for the thermal decomposition of (a) PVC-DINCH23; (b) PVC-DINCH34 and (c) PVC-DINCH45 blends. 

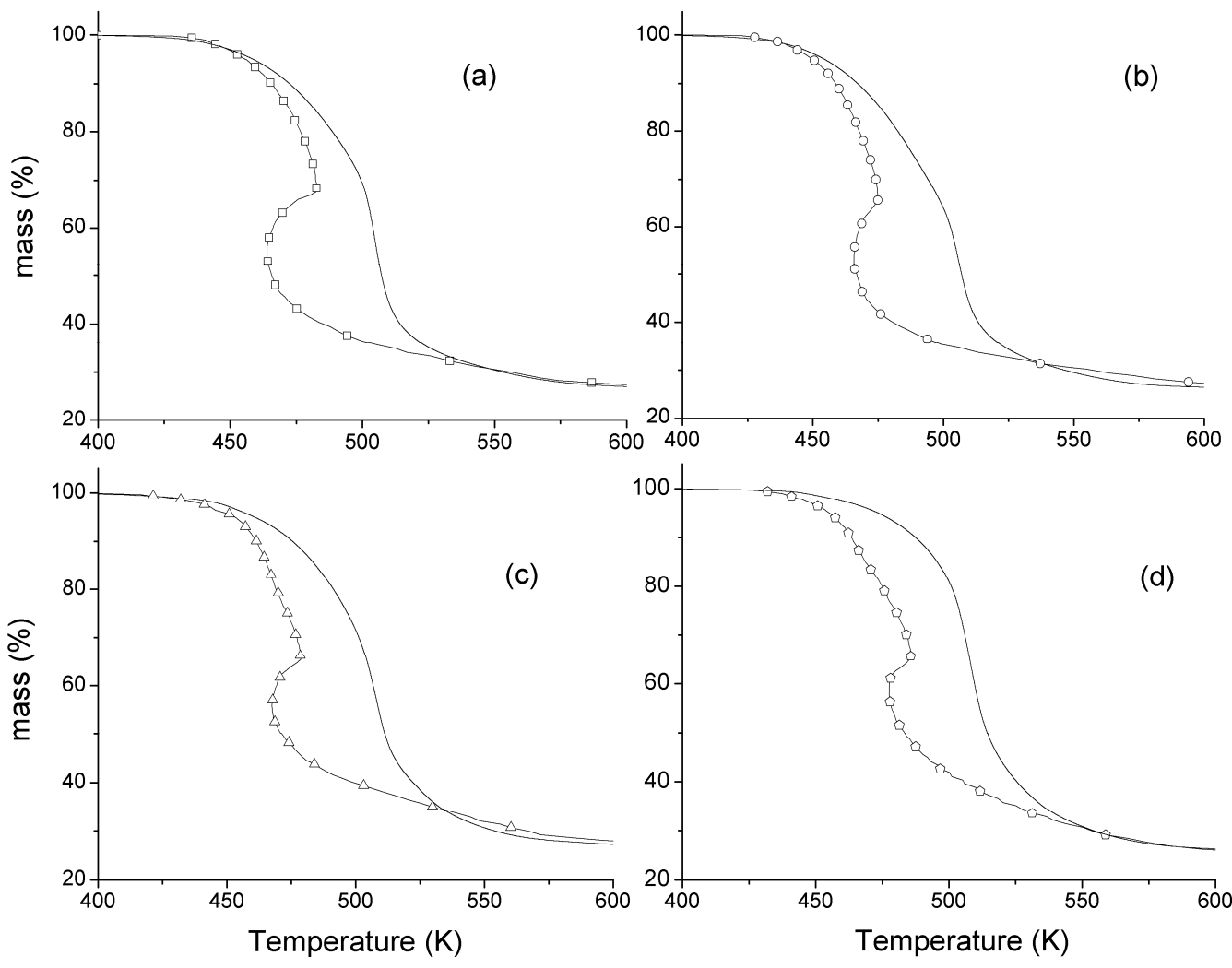

Figure 5. Comparison between experimental mass loss against temperature curves under CRTA and linear heating rate conditions for the thermal decomposition of (a) PVC-DINCH34; (b) PVC-DOP34; (c) PVC-DOTP34 and (d) PVC-DINP34 blends. 
In the introduction, apart from CRTA, two additional sample controlled thermal analysis methods were mentioned: Stepwise Isothermal Analysis (SIA) and High Resolution TGA (Hi-Res). In Figure 6, results obtained from these two methods for the analysis of the PVC-DINCH34 blend are included. Similar experimental times to those of the CRTA experiment in Figures 2 and 3 have been selected for the sake of comparison. In the case of the SIA experiment (Fig. 6a), the temperature increases at a selected heating rate (in the present case $20 \mathrm{~K} \mathrm{~min}^{-1}$ ) up to the point when the mass loss rate reaches a previously set limit value (in this case $0.25 \mathrm{~min}^{-1}$ ). At that point temperature is maintained at a constant level and is not increased again until the reaction rate becomes smaller than the selected limit. ${ }^{48}$ Thus, the experiment proceeds in a series of consecutive linear heating and isothermal events. In the case of the SIA curve for the PVC-DINCH34 blend most of the experiment corresponds to an isotherm at $476 \mathrm{~K}$. Previous studies on the similarities and differences between CRTA and SIA experiments have concluded that for nucleation and growth kinetic models (which is the case of the PVC dehydrochlorination reaction), the SIA curves result in a very long isothermal step followed by a much shorter stepwise increase of temperature up to the completion of the reaction. ${ }^{42}$ That characteristic shape is clearly shown in Figure 6a. Therefore, it is amply clear from this figure that the SIA curve does not allow for discriminating the release of the plasticizer from the dehydrochlorination of PVC and that, in the present case, SIA does not present more resolving power than conventional linear heating rate analysis. Alternatively, in the Hi-Res experiment (Fig. 6b), the heating rate is automatically modified by the instrument following an exponential function of the actual mass loss rate. ${ }^{42-43}$ Thus, during the mass loss events, the heating rate decreases up to minimum value; while in-between reaction events, the heating rate increases up to a maximum value. This method provides a better resolving power than conventional linear heating rate for the PVC-DINCH34 blend (Figures 1 and 6), showing two different mass losses. However, the separation of the events is not sufficient to quantify the percentage of the components, unlike the CRTA measurement. In conclusion, it is clear that neither SIA nor Hi-Res methods are able to successfully discriminate the percentages of plasticizer in the blend, while CRTA can. 

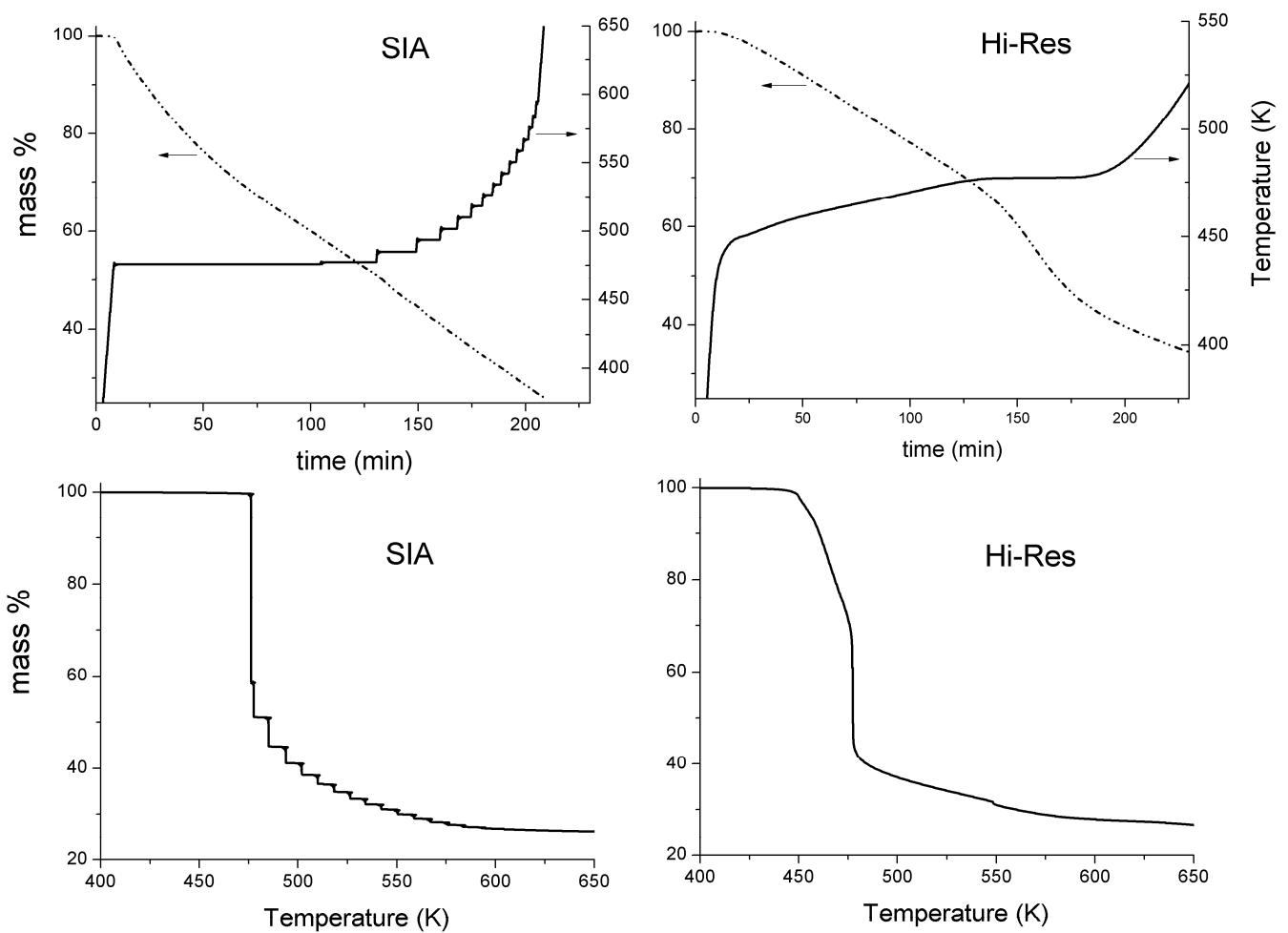

Figure 6. Experimental SIA and Hi-Res TGA curves obtained for the thermal decomposition of the PVC-DINCH34 blend. 


\section{Conclusions}

Here, we have shown the potential of Controlled Rate Thermal Analysis for the compositional characterization of multicomponent polymers. Several PVC-plasticizer blends were used as examples to highlight the higher discrimination power of this technique, which allows for the separation of overlapping steps in a way that cannot be achieved by conventional methods. At the same time, the characteristic shape of the mass-temperature curves obtained under CRTA conditions permits the precise quantification of the plasticizer content in each blend.

\section{ACKNOWLEDGEMENT}

Financial support from projects TEP-03002 from Junta de Andalucía and MAT 200806619/MAT from the Spanish Ministerio de Ciencia e Innovación is acknowledged. 


\section{Bibliography}

(1) Crompton, R. Determination of Additives in Polymers and Rubbers; Rapra, 2007.

(2) Wang, F. C. Y. Journal of Chromatography A. 1999, 843, 413.

(3) Peris-Vicente, J.; Baumer, U.; Stege, H.; Lutzenberger, K.; Adelantado, J. V. G. Analytical Chemistry. 2009, 81, 3180.

(4) Cheremisinoff, N. In Polymer Characterization; Cheremisinoff, N., Ed.; Noyes: Westwood, 1996; pp 1.

(5) Maria, P. C.; Gal, J. F.; Balza, M.; Pere-Trepat, E.; Tumbiolo, S.; Couret, J. M. Analytical Chemistry. 2002, 74, 305.

(6) Bruce Prime, R.; Bair, H. B.; Vyazovkin, S.; Gallagher, P. K.; Riga, A. In Thermal Analysis of Polymers; Bruce Prime, R., Menczel, J. D., Eds.; Wiley, 2009; pp 241.

(7) Chartoff, R. P.; Sircar, A. K. In Encyclopedia of Polymer Science and Technology; John Wiley \& Sons, Inc, 2005.

(8) Daniels, P. H. Journal of Vinyl \& Additive Technology. 2009, 15, 219.

(9) Rahman, M.; Brazel, C. S. Progress in Polymer Science. 2004, 29, 1223.

(10) Handbook of Vinyl Formulating, Second Edition; Grossman, R. F., Ed.; John Wiley \& Sons, Inc.: Hoboken, New Jersey, 2008.

(11) Hakkarainen, M. Chromatography for Sustainable Polymeric Materials: Renewable, Degradable and Recyclable. 2008, 211, 159.

(12) Agarwal, D. K.; Lawrence, W. H.; Turner, J. E.; Autian, J. Journal of Toxicology and Environmental Health. 1989, 26, 39. 
(13) Grasso, P.; Heindel, J. J.; Powell, C. J.; Reichert, L. E. Biology of Reproduction. 1993, 48, 454.

(14) Treinen, K. A.; Heindel, J. J. Reproductive Toxicology. 1992, 6, 143.

(15) Tsutsui, T.; Watanabe, E.; Barrett, J. C. Carcinogenesis. 1993, 14, 611.

(16) Biedermann, M.; Fiselier, K.; Grob, K. Trends in Food Science \& Technology. 2008, 19, 145.

(17) Bodor, S.; Zook, J. M.; Lindner, E.; Toth, K.; Gyurcsanyi, R. E. Analyst. 2008, 133, 635.

(18) Ekelund, M.; Azhdar, B.; Hedenqvist, M. S.; Gedde, U. W. Polymer Degradation and Stability. 2008, 93, 1704.

(19) Lardjane, N.; Belhaneche-Bensemra, N. Journal of Applied Polymer Science. 2009, 111, 525.

(20) Marcilla, A.; Garcia, S.; Garcia-Quesada, J. C. Polymer Testing. 2008, 27, 221.

(21) Monroy, E.; Wolff, N.; Ducruet, V.; Feigenbaum, A. Analusis. 1993, 21, 221.

(22) Rothenbacher, T.; Schwack, W. Rapid Communications in Mass Spectrometry. 2009, 23, 2829.

(23) Wang, Q.; Storm, B. K. Polymer Testing. 2005, 24, 290.

(24) Parreira, T. F.; Ferreira, M. M. C.; Sales, H. J. S.; de Almeida, W. B. Applied Spectroscopy. 2002, 56, 1607.

(25) Jimenez, A.; Iannoni, A.; Torre, L.; Kenny, J. M. Journal of Thermal Analysis and Calorimetry. 2000, 61, 483.

(26) Jimenez, A.; Torre, L.; Kenny, J. M. Polymer Degradation and Stability. 2001, 73, 447.

(27) Jimenez, A.; Lopez, J.; Torre, L.; Kenny, J. M. Journal of Applied Polymer Science. 1999, 73, 1069. 
(28) Benes, M.; Milanov, N.; Matuschek, G.; Kettrup, A.; Placek, V.; Balek, V. Journal of Thermal Analysis and Calorimetry. 2004, 78, 621.

(29) Benes, M.; Placek, V.; Matuschek, G.; Kettrup, A.; Gyoryova, K.; Balek, V. Journal of Applied Polymer Science. 2006, 99, 788.

(30) Parkes, G. M. B.; Barnes, P. A.; Charsley, E. L. Analytical Chemistry. 1999, 71, 2482.

(31) Criado, J. M.; Gotor, F. J.; Ortega, A.; Real, C. Thermochimica Acta. 1992, 199, 235.

(32) Sorensen, O. T.; Rouquerol.J. Sample Controlled Thermal Analysis; Kluwer: Budapest, 2003; Vol. 3.

(33) Llewellyn, P.; Rouquerol, J. Journal of Thermal Analysis and Calorimetry. 2003, 72, 1099.

(34) Koga, N.; Kimizu, T. Journal of the American Ceramic Society. 2008, 91, 4052.

(35) Perez-Maqueda, L. A.; Criado, J. M.; Subrt, J.; Real, C. Catalysis Letters. 1999, $60,151$.

(36) Rouquerol, J. Journal of Thermal Analysis and Calorimetry. 2003, 72, 1081.

(37) Criado, J. M.; Perez-Maqueda, L. A.; Dianez, M. J.; Sanchez-Jimenez, P. E. Journal of Thermal Analysis and Calorimetry. 2007, 87, 297.

(38) Criado, J. M.; Perez-Maqueda, L. A. Journal of Thermal Analysis and Calorimetry. 2005, 80, 27.

(39) Sanchez-Jimenez, P. E.; Perez-Maqueda, L. A.; Perejon, A.; Criado, J. M. Polymer Degradation and Stability. 2009, 94, 2079.

(40) Sanchez-Jimenez, P. E.; Perez-Maqueda, L. A.; Perejon, A.; Criado, J. M. Polymer Degradation and Stability. 2010, 95, 733.

(41) Sorenson, O. T. Journal of Thermal Analysis and Calorimetry. 1999, 56, 17. 
(42) Gotor, F. J.; Perez-Maqueda, L. A.; Ortega, A.; Criado, J. M. J. Therm. Anal. Calorim. 1998, 53, 389.

(43) Gill, P. S.; Sauerbrunn, S. R.; Crowe, B. S. Journal of Thermal Analysis. 1992, 38, 255.

(44) Sauerbrunn, S.; Gill, P. Am Lab. 1994, 26.

(45) Sanchez-Jimenez, P. E.; Criado, J. M.; Perez-Maqueda, L. A. Journal of Thermal Analysis and Calorimetry. 2008, 94, 427.

(46) Sanchez-Jimenez, P. E.; Perejon, A.; Criado, J. M.; Dianez, M. J.; PerezMaqueda, L. A. Polymer. 2010, 51, 3998.

(47) Criado, J. M.; Perez Maqueda, L. A. In Sample Controlled Thermal Analysis:

Origin, Goals, Multiple Forms, Applications and Future; Sorensen, O. T., Rouquerol, J., Eds.; Kluwer: Dordrecht, 2003; Vol. 3; pp 55.

(48) Sorensen, O. T. Journal of Thermal Analysis and Calorimetry. 2003, 72, 1075. 


\section{For TOC only}

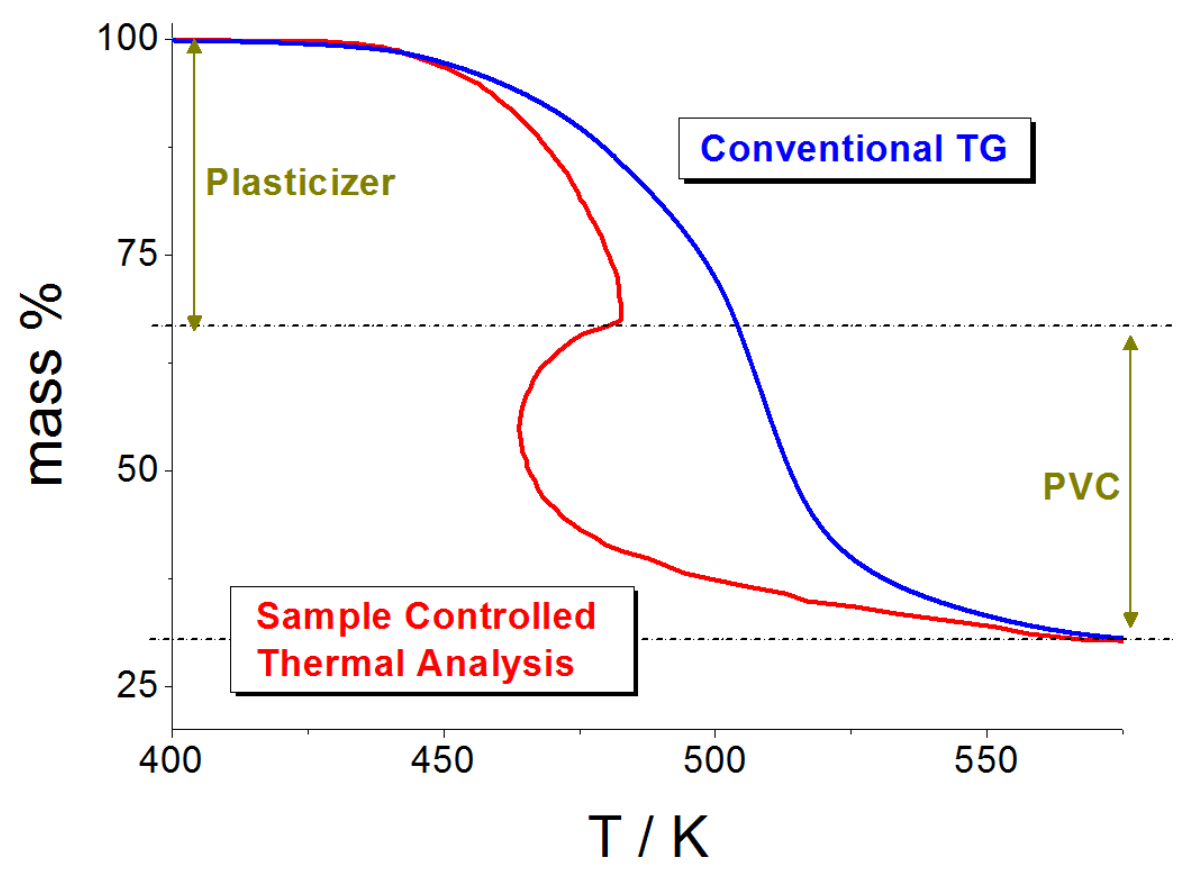


